\title{
The finite strip method for acoustic and vibroacoustic problems
}

\author{
J. Poblet-Puig*, A. Rodríguez-Ferran ${ }^{\dagger}$ \\ Laboratori de Càlcul Numèric \\ E.T.S. d'Enginyers de Camins, Canals i Ports de Barcelona \\ Universitat Politècnica de Catalunya
}

November 30, 2012

\begin{abstract}
The finite strip method, widely employed in structural mechanics, is extended to solve acoustic and vibroacoustic problems. The acoustic part of the formulation, including how to handle the most typical acoustic boundary conditions and the fluid structure interaction, is presented. Several realistic problems where the three-dimensional domain of interest has extrusion symmetry are solved. These examples illustrate the advantages of the method: it has smaller computational costs than the finite element method and consequently the analysed frequency range can be increased.
\end{abstract}

\section{Introduction}

The finite strip method (FSM) has been widely applied to structural analysis during the past four decades [1,2]. This numerical technique can be used for problems whose geometry is constant along a coordinate axis and can be generated by extrusion of a planar face. Some bridge platforms are the classical example where a geometrically complex cross-section can be extruded along the bridge axis in order to generate the whole geometry.

The main idea of the method is to use different interpolation functions at crosssection level and along the extrusion direction. Piecewise functions with local support that can be adapted to the geometrical complexity are used at the cross-section level. On the contrary, global support functions are used along the extrusion direction. This

*correspondence: UPC, Campus Nord B1, Jordi Girona 1, E-08034 Barcelona, Spain, e-mail: jordi.poblet@upc.edu

†e-mail: antonio.rodriguez-ferran@upc.edu 
significantly decreases the computational burden because fewer functions are needed in the extrusion direction and three-dimensional problems can be solved with a cost similar to two-dimensional problems.

Various aspects can break the extrusion symmetry of the problem. Different boundary conditions can be reproduced by means of an adequate choice of the functions used in the extrusion direction. Forces that do not satisfy the extrusion symmetry can be considered by an adequate integration of the force term.

The use of discretisation techniques such as the finite element method (FEM) or the boundary element method (BEM) for acoustic and vibroacoustic problems is often limited by high computational costs. These costs are caused by the fine discretisations required in order to properly describe the pressure or vibration solution fields. The wave length of the solution fields decreases for higher frequencies. This is the reason why the FEM and the BEM are regarded as low-frequency modelling techniques.

The issue of computational costs becomes more important when dealing with threedimensional geometries. In those cases, the number of degrees of freedom drastically increases. However, many problems in acoustics and vibroacoustics have a simple geometry. This geometry can often be generated by extrusion. This is the case of building acoustics where rooms almost always have more than one possible extrusion direction and walls have a geometrically complex cross-section that is constant along a direction.

For these reasons, the finite strip method can be viewed as a valid numerical technique for three-dimensional acoustic and vibroacoustic problems with simple, but usual in real life, geometries. The computational costs of the FSM are smaller than the costs of a three-dimensional FEM calculation.

This geometric extrusion symmetry has been also exploited in vibration analyses for structures that clearly have one dimension that is larger than the others (beams or large rectangular plates for example). The vibration field is obtained by means of the waveguide finite element method (WFEM). Details on the formulation can be found in $[3,4]$. The derivation of the waveguide finite elements is based on Hamilton's principle. They have been considered with several purposes: (i) obtain the vibration field in infinite waveguides; (ii) describe dispersion curves of several structural typologies [5, 6]; (iii) calculate the eigenmodes of the structure and afterwards use them to perform a modal analysis [7].

The WFEM has also been used for fluid-structure interaction problems in waveguides. In [8] the waveguide idea was used to develop a finite element based model. The transmission of sound from the interior of rectangular air filled ducts was studied. The ducts were considered unbounded and a mode-shaped pressure field was imposed in the interior. This was then coupled with the structure modelled with plate elements (without in-plane stiffness). The radiation into infinite acoustic domains was taken into account by means of an intermediate layer of quadrangular acoustic waveguide elements and a set of radiation eigensolutions from a cylindrical surface. The waveguide finite elements are important here in order to perform the transition at cross-section level from a rectangular shaped contour to a circular surface. By imposing the shape of the internal pressure field (and assuming one-way coupling from the interior to the exterior), this model enforces all the pressure and displacement fields to have the same wave number along the extrusion direction. The generalisation of this hypothesis to 
all transmission problem types would implie to assume that only forced transmission occurs. In sound transmission problems, and especially in building acoustics, it is also important to account for resonant transmission. This also includes eigenmodes in the extrusion direction. The interaction between pressure and vibration waves with different wave number along the extrusion direction or that are dephased due to the problem dimensions and boundary conditions can also be relevant and should be considered.

A similar idea is used in [9] in order to generate a set of eigensolutions along the unbounded extrusion direction. This is afterwards used to obtain frequencydependent solutions by means of a modal superposition procedure. The interaction between absorbing materials and air is also considered.

The fluid and fluid-structure interaction elements proposed in [10] are based on a fluid velocity potential formulation. This model has been applied to the calculation of the dispersion curves of water filled steel pipes.

All the works reviewed here coincide in the use of trigonometric functions in the extrusion direction in order to reduce the computational cost of the problem. However, the main difference between the FSM developed in [1] for structural analysis and the WFEM concept used with dynamic and acoustic purposes in $[3-8,10]$ is the way how the problem is solved. In the first case, the interest is focused on structures of finite dimensions and with specific boundary conditions in the extrusion direction. The finite strip elements are used in order to interpolate the displacement field. This field can be enriched by reducing the element size or increasing the number of functions (sines, cosines,...) considered. Both strategies lead to an increase of the number of elements. Afterwards, the weak form of the problem is used in order to derive the systems of linear equations to be solved. The unknowns are contributions to the solution field without a clear physical meaning before their combination (postprocess). In the second case, some assumptions are needed in order to derive the element formulation. In most of the cases a wave length of the solution along the extrusion direction is imposed. Moreover, the extrusion direction is often assumed to be infinite. This hypothesis usually reduces the size of the systems of linear equations to be solved because only one possible wave in the extrusion direction is considered. This provides a meaning to the obtained solution (without requiring major postprocesses), but limits the analysis to waveguides. An extension of the WFEM to more general situations would require additional procedures and the above mentioned advantages would be lost.

In this paper a finite strip formulation is presented for the fluid-pressure vibroacoustic problem. This falls within the first of the above mentioned tendencies. The structural part of the problem is solved according to [1] and the interest is focused here in the acoustic part and the fluid-structure interaction. The vibroacoustic problem is solved considering finite dimensions of the domains and without imposing a wave length along the extrusion direction (pressure or vibration fields) or performing similar assumptions. This is important for the examples shown here, which are oriented to the field of building acoustics and where the resonant response is always relevant. The method is used in order to obtain the frequency-dependent response or the eignfrequencies of the analysed systems. Special attention is paid to the most typical boundary conditions used in building acoustics such as: (i) imposed normal 
velocities; (ii) absorbing boundaries (Robin boundary conditions); (iii) fluid-solid interaction.

The outline of the paper is as follows. The FSM formulation of the pressuredisplacement vibroacoustic equations is shown in Section 2. First the FSM formulation of the acoustic problem is presented in Sections 2.2 and 2.3. Afterwards, the coupling of acoustic domains with elastic structures is developed in Sections 2.4 and 2.5. The application examples are shown in Section 3 and include (i) frequency response of acoustic domains in Section 3.1 and (ii) vibroacoustic problems in Section 3.2. All of them are in the field of building acoustics. The paper is closed with the conclusions of Section 4.

\section{Formulation}

\subsection{The vibroacoustic problem}

The developments, discussion and the examples shown here are based on the steady harmonic pressure-displacement vibroacoustic equations [11, 12]:

Acoustic domain:

$$
\begin{array}{lrl}
\triangle p(\boldsymbol{x})+k^{2} p(\boldsymbol{x})=-\sum_{m=1}^{n_{p s}} \mathrm{i} \omega \rho_{\mathrm{F}} q_{m} \delta\left(\boldsymbol{x}_{m}, \boldsymbol{x}\right) & & \text { in } \Omega_{F} \\
\frac{\partial p(\boldsymbol{x})}{\partial n}=-\mathrm{i} \rho_{\mathrm{F}} \omega v_{n} & & \text { on } \Gamma_{N} \\
\frac{\partial p(\boldsymbol{x})}{\partial n}=-\mathrm{i} \rho_{\mathrm{F}} \omega A p(\boldsymbol{x}) & & \text { on } \Gamma_{R} \\
\frac{\partial p(\boldsymbol{x})}{\partial n}=\rho_{\mathrm{F}} \omega^{2}(\boldsymbol{u}(\boldsymbol{x}) \cdot \boldsymbol{n}) & & \text { on } \Gamma_{F S}
\end{array}
$$

Solid domain:

$$
\begin{array}{lr}
\nabla \cdot \boldsymbol{\sigma}(\boldsymbol{x})=-\rho_{\mathrm{S}} \omega^{2} \boldsymbol{u}(\boldsymbol{x}) & \text { in } \Omega_{S} \\
\boldsymbol{\sigma}(\boldsymbol{x})=\mathbf{C}: \varepsilon & \text { in } \Omega_{S} \\
\boldsymbol{\sigma}(\boldsymbol{x}) \cdot \boldsymbol{n}=\boldsymbol{t}(\boldsymbol{x}, t) & \text { on } \Gamma_{N}^{s} \\
\boldsymbol{u}(\boldsymbol{x})=\boldsymbol{u}(\boldsymbol{x})_{D} & \text { on } \Gamma_{D}^{s} \\
\boldsymbol{\sigma}(\boldsymbol{x}) \cdot \boldsymbol{n}=-p(\boldsymbol{x}) \boldsymbol{n} & \text { on } \Gamma_{F S}
\end{array}
$$

The acoustic part of the problem in the domain $\Omega_{F}$ is governed by the Helmholtz equation (1). $p(\boldsymbol{x})$ is the phasor of acoustic pressure, $k$ is the wave number $(k=\omega / c=$ $2 \pi f / c$, where $f$ is the frequency, $\omega$ the angular frequency and $c$ the speed of sound in the fluid of density $\left.\rho_{\mathrm{F}}\right)$. The presence of $n_{p s}$ point sound sources at positions $\boldsymbol{x}_{m}$ with source strengths $q_{m}$ is considered $\left(\left[q_{m}\right]=\right.$ volume/time $) . v_{n}$ is the velocity imposed on the Neumann contour $\Gamma_{N}$ and $A$ the admittance of the Robin contour $\Gamma_{R}$. This models the acoustic absorption.

The solid domain $\Omega_{S}$ is usually considered as a linear elastic solid. $\boldsymbol{u}(\boldsymbol{x})$ is the phasor of solid displacement, $\boldsymbol{\sigma}$ is the Cauchy stress tensor, $\rho_{\mathrm{S}}$ is the density of the solid, $t$ is the traction vector (forces acting on the solid contour $\Gamma_{N}^{s}$, according to 
Eq. (7)) and $\boldsymbol{u}(\boldsymbol{x})_{D}$ are the imposed displacements in the solid contour $\Gamma_{D}^{s}$. The strain of the solid is: $\varepsilon=\nabla^{s} \boldsymbol{u}(\boldsymbol{x})=\frac{1}{2}\left(\nabla \boldsymbol{u}(\boldsymbol{x})^{T}+\boldsymbol{u}(\boldsymbol{x}) \nabla^{T}\right) . \boldsymbol{n}$ is the exterior normal for every domain.

Continuity of normal velocities and pressures on the interface $\Gamma_{F S}$ is imposed in order to couple the solid and fluid domains.

The numerical formulation of this problem by means of the FEM or the BEM is reviewed in [13, 14]. Various applications of technological interest can be found in the literature. The sound transmission of walls has been studied by means of the FEM in [15] and the BEM in [16]. Very often the applications of numerical techniques in building acoustics are limited to the low-frequency range (due to the large computational costs) or avoid the modelling of the whole problem by means of simplifications (i.e. radiation of sound in unbounded domains).

\subsection{The finite strip method for the Helmholtz equation}

By applying the usual weighted residual approach, the strong form (1) is transformed into the weak form

$$
\begin{aligned}
\int_{\Omega_{F}} \nabla p \cdot \nabla \varphi d \Omega+\int_{\Gamma_{R}} \mathrm{i} \rho_{\mathrm{F}} \omega A p \varphi d \Gamma- & \int_{\Omega_{F}} k^{2} p \varphi d \Gamma= \\
& \int_{\Omega_{F}} \mathrm{i} \omega \rho_{\mathrm{F}} \sum_{m=1}^{n_{p s}} q_{m} \delta\left(\boldsymbol{x}, \boldsymbol{x}_{m}\right) \varphi d \Omega-\int_{\Gamma_{N}} \mathrm{i} \rho_{\mathrm{F}} \omega v_{n} \varphi d \Gamma
\end{aligned}
$$

where $\varphi$ is the test function. This is our starting point in order to obtain the finite strip formulation for an acoustic domain with extrusion symmetry such as the one shown in Fig. 1. Capital letters are used in order to identify global coordinates/displacements while small letters are used for the local variables (in the finite elements). $Z=z$ is the coordinate assigned to the extrusion direction.

The pressure field is interpolated by means of strip functions. These can be understood as standard FEM interpolation functions $N_{j}(x, y)[17]$ in the $X Y$ plane, multiplied by appropriate interpolation functions $\mathcal{Z}_{s}(z)$ in the $z$ direction

$$
p(\boldsymbol{x})=\sum_{s=0}^{n_{Z}^{F}} \sum_{j=1}^{n_{\text {nodes }}^{F}} N_{j}(x, y) \mathcal{Z}_{s}(z) p_{j, s} \quad ; \quad p_{j, s} \in \mathbb{C} \quad ; \quad N_{j}: \mathbb{R}^{2} \rightarrow \mathbb{R}
$$

$n_{\text {nodes }}^{F}$ is the number of nodes in the cross-section (i.e. for constant $\left.z\right)$. $\left(n_{Z}^{F}+1\right)$ is the number of interpolation functions in the $z$ direction. $p_{j, s}$ is the pressure phasor value at node $j$ for the interpolation function $\mathcal{Z}_{s}(z)$. $\mathcal{Z}_{s}(z)$ must satisfy the boundary conditions at the limit cross-sections $Z=Z_{0}$ and $Z=Z_{L}$. Note that, in the FSM, $\left(n_{Z}^{F}+1\right)$ sets of $n_{\text {nodes }}^{F}$ nodal values each are required. Due to the Kronecker delta property of the interpolation functions used, in the FEM $p_{j}$ is directly the nodal pressure value. A different situation is found in the FSM, where in order to obtain the $j^{\text {th }}$ nodal value of pressure at each cross-section of constant $z$ a combination of the values $p_{j, s}$ is needed:

$$
p_{j}=\sum_{s=0}^{n_{Z}^{F}} \mathcal{Z}_{s}(z) p_{j, s}
$$




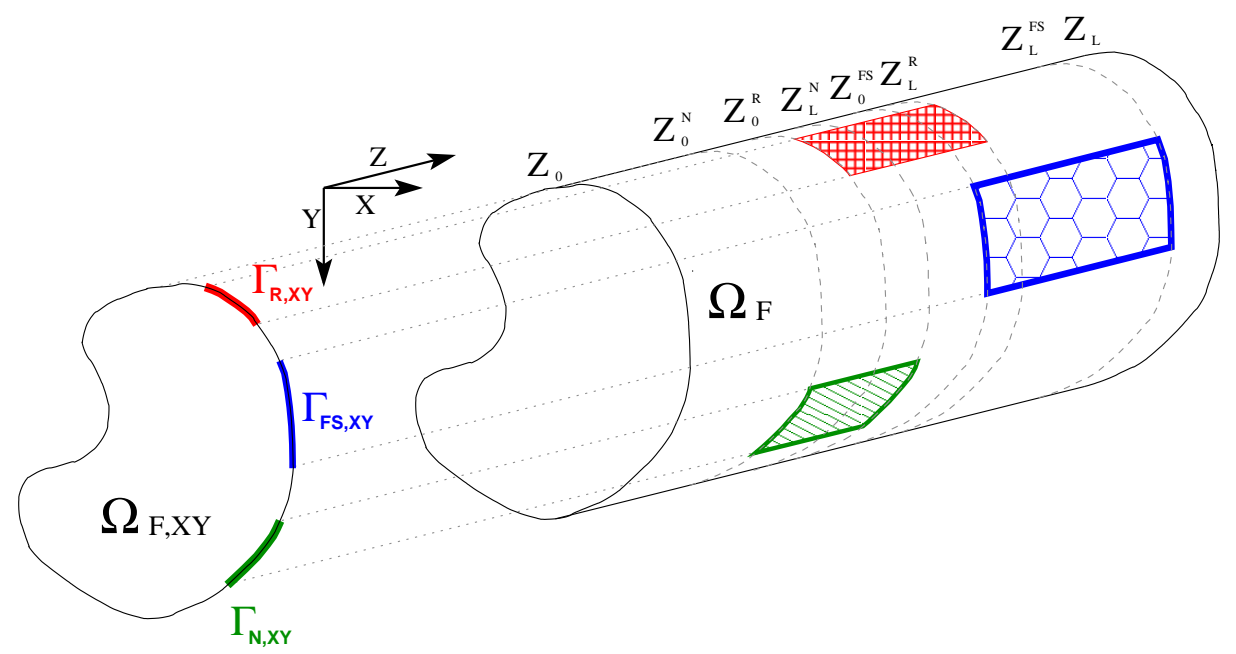

Figure 1: Sketch and notation used in the FSM formulation of an acoustic domain that can be generated by extrusion along the $z$ direction. On the left, the two-dimensional computational domain. On the right the three-dimensional physical domain. The colored and patterned regions represent several boundary conditions for the fluid domain: $\Gamma_{R}$ (red), the absorbing contour modelled with a Robin boundary condition; $\Gamma_{N}$ (green), the contour where a normal velocity is imposed; $\Gamma_{F S}$ (blue), the region that is in contact with a vibrating solid. $Z_{0}^{\square}$ and $Z_{L}^{\square}$ denote the beginning and ending of each region along the extrusion direction.

The test functions $\varphi$ are chosen of the same type as the interpolation functions of Eq. (11):

$$
\varphi(\boldsymbol{x})=\sum_{r=0}^{n_{Z}^{F}} \sum_{i=1}^{n_{\text {nodes }}^{F}} N_{i}(x, y) \mathcal{Z}_{r}(z) \varphi_{i, r} \quad ; \quad \varphi_{i, r} \in \mathbb{C} \quad ; \quad N_{i}: \mathbb{R}^{2} \rightarrow \mathbb{R}
$$

Eq. (10) must be satisfied for any set of values $\varphi_{i, r}$.

With the proposed interpolation and test functions the operations in the weak form of Eq. (10) can be split into the ones in the $X Y$ cross-section and the ones in the $z$ direction. The $z$ direction will be treated analytically in order to reduce the problem to a two-dimensional FEM calculation in the $X Y$ cross-section. Thus, $\left(n_{Z}^{F}+1\right)$ sets of $n_{\text {nodes }}^{F}$ linear equations are obtained.

As an example, consider the first term of the weak form of Eq. (10) for the test function $\varphi=N_{i}(x, y) \mathcal{Z}_{r}(z)$. The three-dimensional Laplacian contribution to the $i, j$ matrix coefficient corresponding to the strip functions $s$ and $r$ can be written as

$$
\begin{aligned}
& {\left[\int_{\Omega_{F}} \nabla p \cdot \nabla \varphi d \Omega\right]_{i, j ; r, s}=\int_{\Omega_{F, X Y}}\left(\nabla_{X Y} N_{i} \cdot \nabla_{X Y} N_{j}\right) d x d y \int_{Z_{0}}^{Z_{L}} \mathcal{Z}_{r}(z) \mathcal{Z}_{s}(z) d z+} \\
+ & \int_{\Omega_{F, X Y}} N_{i} N_{j} d x d y \int_{Z_{0}}^{Z_{L}} \mathcal{Z}_{r}^{\prime}(z) \mathcal{Z}_{s}^{\prime}(z) d z \quad i, j=1,2, \ldots, n_{\text {nodes }}^{F} \quad r, s=0,1,2, \ldots, n_{Z}^{F}
\end{aligned}
$$


where $\mathcal{Z}_{r}^{\prime}(z)=d \mathcal{Z}_{r}(z) / d z, \nabla_{X Y}$ is the gradient in the $X Y$ plane, $\Omega_{F, X Y}$ is the twodimensional cross-section in the $X Y$ plane. $Z_{0}$ and $Z_{L}$ are the limits of integration for the acoustic domain $\Omega_{F}$ as shown in Fig. 1. A similar substitution can be done for the other terms of Eq. (10) in order to obtain

$$
\left[\begin{array}{ccc}
\mathbf{A}_{0,0}^{F} & \cdots & \mathbf{A}_{0, n_{Z}^{F}}^{F} \\
\vdots & \ddots & \vdots \\
\mathbf{A}_{n_{Z}^{F}, 0}^{F} & \cdots & \mathbf{A}_{n_{Z}^{F}, n_{Z}^{F}}^{F}
\end{array}\right]\left\{\begin{array}{c}
\mathbf{p}_{0} \\
\vdots \\
\mathbf{p}_{n_{Z}^{F}}
\end{array}\right\}=\left\{\begin{array}{c}
\mathbf{f}_{0}^{F} \\
\vdots \\
\mathbf{f}_{n_{Z}^{F}}^{F}
\end{array}\right\}
$$

with

$$
\mathbf{A}_{r, s}^{F}=\left[\mathbf{K}_{X Y}^{F} I_{1}(r, s)+c^{2} \mathbf{M}_{X Y}^{F} I_{2}(r, s)\right]+\mathrm{i} \omega \mathbf{C}_{X Y}^{F} I_{R}(r, s)-\omega^{2} \mathbf{M}_{X Y}^{F} I_{1}(r, s)
$$

and where

$$
\begin{aligned}
& I_{1}(r, s)=\int_{Z_{0}}^{Z_{L}} \mathcal{Z}_{r}(z) \mathcal{Z}_{s}(z) d z \\
& I_{2}(r, s)=\int_{Z_{0}}^{Z_{L}} \mathcal{Z}_{r}^{\prime}(z) \mathcal{Z}_{s}^{\prime}(z) d z \\
& I_{R}(r, s)=\int_{Z_{0}^{R}}^{Z_{L}^{R}} \mathcal{Z}_{r}(z) \mathcal{Z}_{s}(z) d z
\end{aligned}
$$

and $\mathbf{M}_{X Y}^{F}, \mathbf{C}_{X Y}^{F}, \mathbf{K}_{X Y}^{F}$ are the two-dimensional mass, absorption and stiffness matrices defined as

$$
\begin{aligned}
\left(\mathbf{K}_{X Y}^{F}\right)_{i j} & =\int_{\Omega_{F, X Y}} \nabla N_{i} \cdot \nabla N_{j} d \Omega_{F, X Y} \\
\left(\mathbf{C}_{X Y}^{F}\right)_{i j} & =\int_{\Gamma_{R, X Y}} \rho_{\mathrm{F}} A N_{i} N_{j} d \Gamma \\
\left(\mathbf{M}_{X Y}^{F}\right)_{i j} & =\frac{1}{c^{2}} \int_{\Omega_{F, X Y}} N_{i} N_{j} d \Omega_{F, X Y}
\end{aligned}
$$

The expression of the acoustic force vector is

$$
\begin{aligned}
\left(\mathbf{f}_{r}^{F}\right)_{i}=\mathrm{i} \rho_{\mathrm{F}} \omega \sum_{m=1}^{n_{p s}} \int_{\Omega_{F, X Y}} q_{m} \delta_{\left(x_{m}, y_{m}\right)} N_{i} d \Omega_{F, X Y} \int_{Z_{0}}^{Z_{L}} \mathcal{Z}_{r}(z) \delta_{z_{m}} d z & \\
& -\sum_{j=1}^{n_{v}} \int_{\Gamma_{n, X Y}} \mathrm{i} \rho_{\mathrm{F}} \omega N_{i} N_{j}^{v} d \Gamma_{x, y} \int_{Z_{0}^{N}}^{Z_{L}^{N}} \mathcal{Z}_{r}(z) v_{n}(z)_{j} d z
\end{aligned}
$$

where the imposed velocity on the $\Gamma_{N}$ boundary is

$$
v_{n}(x, y, z)=\sum_{j=1}^{n_{v}} N_{j}^{v}(x, y) v_{n}(z)_{j}
$$

and $N_{j}^{v}$ are interpolation functions restricted to the boundary. $n_{v}$ is the number of nodes with imposed velocity. 
Eq. (15) shows the block structure of the system of linear equations to be solved. Each block is related with the strip functions $r$ and $s$, while the indices $i$ and $j$ inside the block are related with the discretisation in the $X Y$ plane. The off-diagonal blocks are zero if the strip functions are orthogonal. This is an important aspect of the method. It will be more efficient from the computational point of view to solve $n_{Z}^{F}+1$ smaller linear systems of equations than a large linear system including all the blocks.

\subsection{Acoustic strip functions}

The interpolation functions $\mathcal{Z}_{r}(z)$ in the $z$ direction highly influence the FSM performance and the precision of the obtained solutions. Two issues have to be considered. On the one hand, $\mathcal{Z}_{r}(z)$ must satisfy the boundary conditions at $z=Z_{0}$ and $z=Z_{L}$. On the other hand, the group of functions must be complete enough to reproduce the solution variation along the $z$ axis.

An interesting, but not necessary, property to be satisfied is orthogonality. If the integrals shown in Eqs. (17), (18) and (19) vanish for $r \neq s$ the blocks in Eq. (15) become uncoupled.

Several strip functions depending on the boundary conditions are proposed in [1] for the case of structural analysis. Here their equivalent for acoustics and the Helmholtz equation are shown.

The simplest option is to find solutions of

$$
\mathcal{Z}_{r}^{\prime \prime}(z)+k_{r}^{2} \mathcal{Z}_{r}(z)=0
$$

when the contours at $z=Z_{0}$ and $z=Z_{L}$ are purely reflecting. The boundary conditions to satisfy are then

$$
\frac{\partial p(\boldsymbol{x})}{\partial n}=0 \quad \text { on } z=Z_{0} \text { and } z=Z_{L}
$$

and the set of one-dimensional interpolation functions that satisfies Eq. (26) in the $z$ direction

$$
\mathcal{Z}_{r}(z)=\cos \left(\frac{r \pi\left(z-Z_{0}\right)}{\left(Z_{L}-Z_{0}\right)}\right) \quad r=0,1,2, \ldots
$$

with $k_{r}=r \pi /\left(Z_{L}-Z_{0}\right)$. The cosine functions have the following orthogonality properties:

$$
\begin{array}{r}
I_{1}(r, s)=\int_{Z_{0}}^{Z_{L}} \mathcal{Z}_{r}(z) \mathcal{Z}_{s}(z) d z=\left\{\begin{array}{lr}
0 & \text { if } r \neq s \\
Z_{L}-Z_{0} & \text { if } r=s=0 \\
\left(Z_{L}-Z_{0}\right) / 2 & \text { if } r=s>0
\end{array}\right. \\
I_{2}(r, s)=\int_{Z_{0}}^{Z_{L}} \mathcal{Z}_{r}^{\prime}(z) \mathcal{Z}_{s}^{\prime}(z) d z= \begin{cases}0 & \text { if } r \neq s \\
0 & \text { if } r=s=0 \\
\frac{\pi^{2} r^{2}}{2\left(Z_{L}-Z_{0}\right)} & \text { if } r=s>0\end{cases}
\end{array}
$$

Orthogonality according to the scalar product of Eq. (19) is, in general, not satisfied. Thus, problems with acoustic absorption only in a zone along the extrusion direction have a fully coupled system in Eq. (15). 
The boundary conditions (26) are the more 'friendly' for the Helmholtz equation. Like most of the boundary conditions imposed to a structure (blocked displacements or rotations), they do not depend on the frequency and known expressions of scalar products $I_{1}(r, s)$ and $I_{2}(r, s)$ are available. They are often found in realistic building acoustic models. However, different boundary conditions can also be of interest. This is the case of an acoustic domain with absorbing surfaces at $z=Z_{0}$ and $z=Z_{L}$, modelled by means of Robin boundary conditions

$$
\frac{\partial p(\boldsymbol{x})}{\partial n}=-\mathrm{i} \rho_{\mathrm{F}} \omega A_{0} p(\boldsymbol{x}) \quad \text { on } z=Z_{0} \quad ; \quad \frac{\partial p(\boldsymbol{x})}{\partial n}=-\mathrm{i} \rho_{\mathrm{F}} \omega A_{L} p(\boldsymbol{x}) \quad \text { on } z=Z_{L}
$$

where $A_{0}$ and $A_{L}$ are the admittances in the surfaces at $z=Z_{0}$ and $z=Z_{L}$. The generic solution for the one-dimensional problem composed of Eq. (25) and the onedimensional version of the boundary conditions (30) (projected on the $z$ direction) is of the form

$$
\mathcal{Z}_{r}(z)=C_{1} e^{\mathrm{i} k_{r} z}+C_{2} e^{-\mathrm{i} k_{r} z} \quad k_{r}, C_{1}, C_{2} \in \mathbb{C}
$$

leading to the following system of equations with unknowns $C_{1}$ and $C_{2}$ :

$$
\left[\begin{array}{cc}
k_{r}-\rho_{\mathrm{F}} \omega A_{0} & -k_{r}-\rho_{\mathrm{F}} \omega A_{0} \\
\left(k_{r}+\rho_{\mathrm{F}} \omega A_{L}\right) e^{\mathrm{i} k_{r} \ell_{z}} & \left(-k_{r}+\rho_{\mathrm{F}} \omega A_{L}\right) e^{-\mathrm{i} k_{r} \ell_{z}}
\end{array}\right]\left\{\begin{array}{l}
C_{1} \\
C_{2}
\end{array}\right\}=\left\{\begin{array}{l}
0 \\
0
\end{array}\right\}
$$

This system has non-null solutions only if the determinant of the $2 \times 2$ matrix is equal to zero. For a constant frequency $\omega$ this leads to a root finding problem in the complex plane where the unknown is $k_{r}$. Due to the presence of sines and cosines an infinite set of solutions $k_{r}$ can be found. This makes it possible to generate an infinite series of wave functions $\mathcal{Z}_{r}(z)$ in order to interpolate along the $z$ direction. If the undamped values of the wave number $r \pi /\left(Z_{L}-Z_{0}\right)$ are used as initial guess, the convergence is reached very fast with Newton's root finding method. Some numerical values are provided in Table 1 for the case $\left(Z_{L}-Z_{0}\right)=3.5 \mathrm{~m}$. It can be seen that a damped wave number has a small imaginary part that causes the attenuation.

Table 1: Numerical values of the wave number $k_{r}$ for different values of acoustic absorption $\alpha$.

\begin{tabular}{cccc}
\hline$r$ & 1 & 2 & 3 \\
\hline$r \pi /\left(Z_{L}-Z_{0}\right), \alpha=0 \%$ & 0.897597 & 1.795194 & 2.692791 \\
$k_{r}, \alpha=6 \%$ & $0.905350+0.083184 \mathrm{i}$ & $1.796184+0.042046 \mathrm{i}$ & $2.693086+0.028028 \mathrm{i}$ \\
$k_{r}, \alpha=70 \%$ & $0.759194+0.292140 \mathrm{i}$ & $1.990465+0.481909 \mathrm{i}$ & $2.750129+0.351009 \mathrm{i}$ \\
\hline
\end{tabular}

Two drawbacks should be noted. On the one hand, if the boundary conditions depend on the problem frequency $\omega$ (which is the case of Eq. (30) and acoustic problems in general), the set of functions to interpolate in the symmetry direction must be generated for each frequency. On the other hand the set of functions do not satisfy at the same time orthogonality according to the scalar products in Eqs. (17) and (18). Even if they were orthogonalised with respect to one of the two scalar products by means of a Gram-Schmidt procedure, the resulting set of functions would still be 
non-orthogonal with respect to the other. This can be seen if Eq. (25) is multiplied by $\mathcal{Z}_{s}(z)$ and integrated by parts. The following weak form of the equation is obtained:

$-\int_{Z_{0}}^{Z_{L}} \mathcal{Z}_{r}^{\prime}(z) \mathcal{Z}_{s}^{\prime}(z) d z+k_{r} \int_{Z_{0}}^{Z_{L}} \mathcal{Z}_{r}(z) \mathcal{Z}_{s}(z) d z+\mathcal{Z}_{r}^{\prime}\left(Z_{L}\right) \mathcal{Z}_{s}\left(Z_{L}\right)-\mathcal{Z}_{r}^{\prime}\left(Z_{0}\right) \mathcal{Z}_{s}\left(Z_{0}\right)=0$

This has to be satisfied for any pair of functions $\mathcal{Z}_{r}(z)$ and $\mathcal{Z}_{s}(z)$. The scalar products (17) and (18) appear now in Eq. (33). It can be seen that a set of strip functions $\mathcal{Z}_{r}(z)$ will be orthogonal with respect to both scalar products at the same time if and only if $\mathcal{Z}_{r}^{\prime}\left(Z_{L}\right) \mathcal{Z}_{s}\left(Z_{L}\right)-\mathcal{Z}_{r}^{\prime}\left(Z_{0}\right) \mathcal{Z}_{s}\left(Z_{0}\right)=0$. This only happens for some case such as the homogeneous boundary conditions of Eq. (25).

When this double orthogonality cannot be obtained, the system of equations derived from Eq. (15) is coupled (between blocks $r$ and $s$ ). Some of the off-diagonal blocks are non-null.

\subsection{The vibroacoustic problem: coupling between the FSM acoustic and solid domains}

When the acoustic domain is coupled with a vibrating structure, see Eqs. (4) and (9), an integral in the left-hand-side of the weak formulation of the acoustic problem Eq. (10) must be added. The acoustic force due to the solid vibration is then expressed as

$$
\mathbf{f}^{F S}=\int_{\Gamma_{F S}} \rho_{\mathrm{F}} \omega^{2} \varphi(\boldsymbol{u}(\boldsymbol{x}) \cdot \boldsymbol{n}) d \Gamma
$$

where $\boldsymbol{n}$ is here the outward unit normal with respect to the acoustic domain.

If the FSM is used also for the structure, the displacement field is interpolated as

$$
\boldsymbol{u}(\boldsymbol{x})=\sum_{q=1}^{n_{Z}^{S}} \sum_{j=1}^{n_{\text {nodes }}^{S}} \mathbf{N}_{j}(\boldsymbol{X}) \cdot \mathbf{U}_{j, q}(z)=\sum_{q=1}^{n_{Z}^{S}} \sum_{j=1}^{n_{\text {nodes }}^{S}} \mathbf{N}_{j}(\boldsymbol{x}) \cdot\left(\mathbf{T}^{T} \cdot \mathbf{u}_{j, q} \cdot \mathbf{Z}_{q}(z)\right)
$$

where $n_{Z}^{S}$ is the number of strip functions used for the structure, $n_{\text {nodes }}^{S}$ is the number of solid nodes in the cross-section, $\mathbf{N}_{j}(\boldsymbol{X}) / \mathbf{N}_{j}(\boldsymbol{x})$ are the shape functions in global/local coordinates. $\mathbf{T}$ is a transfer matrix of an Euler beam element but with an additional degree of freedom. $\mathbf{T}^{T}$ transforms from local $(x, y)$ to global $(X, Y)$ coordinates in the $X Y$ plane (keeping variables unmodified in the $z$ direction).

Note that, for the acoustic functions $\mathcal{Z}_{r}(z)$, the subscript $r$ starts at 0 . They are often cosines, see Eq. (27), and the constant function is necessary. On the contrary, the subscript $q$ of the structural functions $\mathbf{Z}_{q}(z)$ starts at 1 . In the out-of-plane displacements, they are often sines. Considering $q=0$ for sines would lead to a null function. The solid strip functions depend on the local coordinates of the structural element because, in general, each local displacement component uses different interpolation along the extrusion direction.

The degrees of freedom considered in each structural node if finite strip shell elements are considered are

$$
\mathbf{U}_{j}=\left[u_{X}, u_{Y}, u_{Z}, \theta_{Z}\right]^{T}=\mathbf{T}^{T} \cdot \mathbf{u}_{j}=\left(\left[u_{x}, u_{y}, u_{z}, \theta_{z}\right] \mathbf{T}\right)^{T}
$$


with $u_{Z}=u_{z}$ and $\theta_{Z}=\theta_{z}$. The local directions in the two nodes shell strip are: $x$, direction defined by the element nodes, contained in the shell plane; $y$, direction orthogonal to the shell; $z=x \wedge y$ (being $\wedge$ the cross product), coincident with the extrusion direction (see Fig. 2). $\mathbf{Z}_{q}(z)$ is a diagonal matrix with the interpolation functions in the $z$ direction used for the structural displacements in local coordinates

$$
\mathbf{Z}_{q}(z)=\left[\begin{array}{cccc}
\mathcal{Z}_{q}^{u_{x}}(z) & 0 & 0 & 0 \\
0 & \mathcal{Z}_{q}^{u_{y}}(z) & 0 & 0 \\
0 & 0 & \mathcal{Z}_{q}^{u_{z}}(z) & 0 \\
0 & 0 & 0 & \mathcal{Z}_{q}^{\theta_{z}}(z)
\end{array}\right]
$$

For the case of structural strips satisfying the boundary conditions (in local coordinates) $u_{x}=0, u_{z}=0, \theta_{z}=0$, as described in [1],

$$
\begin{aligned}
\mathcal{Z}_{q}^{u_{x}}(z)=\mathcal{Z}_{q}^{u_{y}}(z) & =\mathcal{Z}_{q}^{\theta_{z}}(z)=\sin \left(\frac{\pi q\left(z-Z_{0}^{F S}\right)}{Z_{L}^{F S}-Z_{0}^{F S}}\right) \\
\mathcal{Z}_{q}^{u_{z}}(z) & =\cos \left(\frac{\pi q\left(z-Z_{0}^{F S}\right)}{Z_{L}^{F S}-Z_{0}^{F S}}\right)
\end{aligned}
$$

The acoustic nodal forces (component $i$ in the contribution associated with the fluid function $\mathcal{Z}_{r}(z)$ ) caused by the vibroacoustic coupling (the part related with $\mathbf{Z}_{q}(z)$ ) can now be written as

$$
\begin{aligned}
& \left(\mathbf{f}^{F_{r} S_{q}}\right)_{i}=-\rho_{\mathrm{F}} \omega^{2} \sum_{j=1}^{n_{\text {nodes }}^{S}}\left(\int_{\Gamma_{F S}}-N_{i}(\boldsymbol{X}) \boldsymbol{n} \cdot\left(\mathbf{N}_{j}(\boldsymbol{X}) \mathbf{U}_{j}(z)\right) d \Gamma\right)=
\end{aligned}
$$

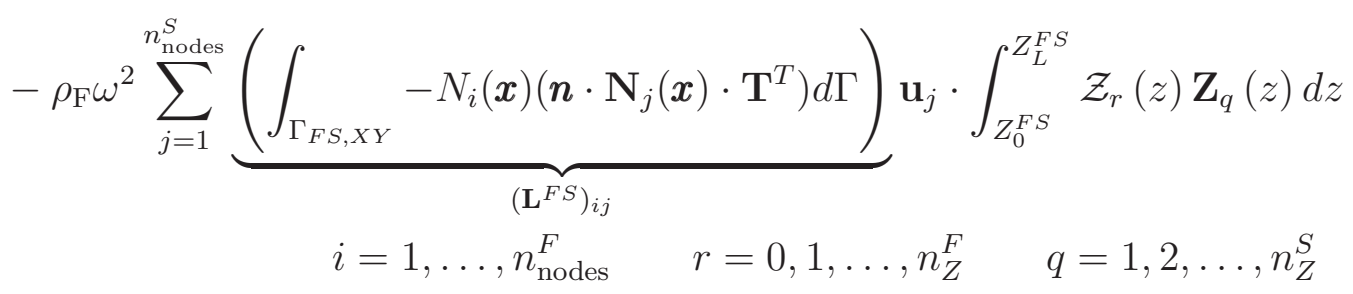

where $\left(\mathbf{L}^{F S}\right)_{i j}$ is the sub-matrix that links the acoustic force in node $i$ with the structural displacements of the solid node $j$. This matrix is calculated in the $X Y$ twodimensional space.

In practice only the normal direction to the boundary is important for fluidstructure coupling because the tangential interaction forces can be neglected for perfect fluids. Thus only the local $y$ component contributes to the coupling force and if $\mathcal{Z}_{q}^{u_{y}}(z)=\mathcal{Z}_{q}^{\theta_{z}}(z)$

$$
\mathbf{f}^{F_{r} S_{q}}=-\rho_{\mathrm{F}} \omega^{2}\left[\int_{Z_{0}^{F S}}^{Z_{L}^{F S}} \mathcal{Z}_{r}(z) \mathcal{Z}_{q}^{u_{z}}(z) d z\right] \quad \mathbf{L}^{F S} \cdot \mathbf{u}_{q}^{g l o b a l}
$$

A similar consideration can be done for the forces caused by the fluid over the structure and it can be written that

$$
\mathbf{f}^{S_{q} F_{r}}=\int_{Z_{0}^{F S}}^{Z_{L}^{F S}} \mathcal{Z}_{r}(z) \mathcal{Z}_{q}^{u_{z}}(z) d z \quad \mathbf{L}^{S F} \cdot \mathbf{p}_{r}
$$


$\mathbf{L}^{S F}$ takes into account the effect of the acoustic fluid over the structure and $\mathbf{L}^{F S}=$ $\left(\mathbf{L}^{S F}\right)^{T}$. It must be noted that $\mathbf{L}^{F S}$ has to be only computed once and then reused to generate all the fluid-structure coupling matrices between the $r$ contribution of fluid $F$ and the $q$ contribution of solid $S$

$$
\mathbf{L}^{F_{r} S_{q}}=\mathbf{L}^{F S} \int_{Z_{0}^{F S}}^{Z_{L}^{F S}} \mathcal{Z}_{r}(z) \mathcal{Z}_{q}^{u_{z}}(z) d z=\mathbf{L}^{F S} I^{F S}(r, q)=\mathbf{L}^{F S} I^{S F}(q, r)
$$

Details on how $\mathbf{L}^{F S}$ is calculated can be found below in Section 2.5.

The system of equations to be solved for the case of a fluid $(F)$ and a solid $(S)$ domain is

$$
\left[\begin{array}{cccccc}
\mathbf{A}_{1,1}^{S} & \ldots & \mathbf{A}_{1, n_{Z}^{S}}^{S} & -\mathbf{L}^{S_{1} F_{0}} & \ldots & -\mathbf{L}^{S_{1} F_{n_{Z}^{F}}} \\
\vdots & \ddots & \vdots & \vdots & \ddots & \vdots \\
\mathbf{A}_{n_{Z}^{S}, 1}^{S} & \ldots & \mathbf{A}_{n_{Z}^{S}, n_{Z}^{S}}^{S} & -\mathbf{L}^{S_{n_{Z}^{S}} F_{0}} & \ldots & -\mathbf{L}^{S_{n_{Z}^{S}} F_{n_{Z}}} \\
\omega^{2} \rho_{\mathrm{F}} \mathbf{L}^{F_{0} S_{1}} & \ldots & \omega^{2} \rho_{\mathrm{F}} \mathbf{L}^{F_{1} n_{Z}} & \mathbf{A}_{0,0}^{F} & \ldots & \mathbf{A}_{0, n_{Z}^{F}}^{F} \\
\vdots & \ddots & \vdots & \vdots & \ddots & \vdots \\
\omega^{2} \rho_{\mathrm{F}} \mathbf{L}^{F_{n_{Z}^{F} S_{1}}} & \ldots & \omega^{2} \rho_{\mathrm{F}} \mathbf{L}^{F_{n_{Z}^{F} S_{n_{Z}}}} & \mathbf{A}_{n_{Z}^{F}, 0}^{F} & \ldots & \mathbf{A}_{n_{Z}^{F}, n_{Z}^{F}}^{F}
\end{array}\right]\left\{\begin{array}{c}
\mathbf{u}_{1} \\
\vdots \\
\mathbf{u}_{n_{Z}} \\
\mathbf{p}_{0} \\
\vdots \\
\mathbf{p}_{n_{Z}^{F}}
\end{array}\right\}=\left\{\begin{array}{c}
\mathbf{f}_{1}^{S} \\
\vdots \\
\mathbf{f}_{n_{Z}^{S}}^{S} \\
\mathbf{f}_{0}^{F} \\
\vdots \\
\mathbf{f}_{n_{Z}^{F}}^{F}
\end{array}\right\}
$$

with

$$
\mathbf{A}_{q, t}^{S}=\mathbf{K}_{q, t}^{S}+\mathrm{i} \omega \mathbf{C}_{q, t}^{S}-\omega^{2} \mathbf{M}_{q, t}^{S}
$$

and $\mathbf{A}_{r, s}^{F}$ the matrix defined in Eq. (16). $\mathbf{M}_{q, t}^{S}, \mathbf{K}_{q, t}^{S}$ and $\mathbf{C}_{q, t}^{S}$ are the mass, stiffness and damping FSM matrices for the solid part of the problem. $\mathbf{f}_{q}^{S}$ is the solid force vector. Details of their formulation for several structural finite strips can be found in [1]. The formulation of these solid matrices is more complex than for the fluid and the contribution of the $z$ direction cannot be separated. If it is a simply supported strip, the solid part matrix $\mathbf{A}^{S}$ in the system of equations (44) is block diagonal. However the global system is, in general, not block diagonal due to the coupling between the fluid and solid. For the case of multiple solids with multiple fluids, the block structure in Eq. (44) must be repeated.

\subsection{Details on the implementation of the coupling procedures}

Different options can be considered in order to implement fluid-structure coupling in finite element softwares. They can be classified into two main groups, depending on the use of congruent or non-congruent meshes.

When congruent meshes are used, there is a univocal relationship between the fluid and solid elements in the fluid-structure interface. Each solid element is in contact with only one fluid element that has the same size. This is an advantage from the implementation point of view. Sometimes the concept of 'coupling element' is used. This can be understood as a layer between the solid and fluid elements with the same connectivity and that is used to calculate the coupling matrices. Two main drawbacks of this congruent mesh option can be mentioned: 
- The element size of the fluid and solid meshes has to be the same. This is not optimal because the wave length in both domains is often different. If the fluid and solid meshes are not adapted to the expected wave length, the computational costs of the problem are larger.

- To have an univocal relationship between the fluid and solid elements is a constraint in the mesh building process. The solid and fluid domains are in practise meshed with a single mesh. It would be easier to mesh each solid and fluid domain independently from the others.

Code-Aster [18] accepts mainly congruent meshes without doubled nodes in its basic operations. In fact, the model properties and formulation have to be assigned to the elements of the mesh and the software decides the variable types of each node (displacements for solid massive elements, displacements and rotations for shell elements, pressure/velocity potential for acoustic elements and all of them in nodes defined in an interface zone). The concept of 'coupling element' is used in [19].

In order to avoid the two drawbacks mentioned above, a non-congruent mesh option can be considered. The additional efforts are related with the implementation. On the one hand, some strategy in order to define the contact zones and identify the elements from the fluid and solid sides must be established. On the other hand, functions that transmit the information between the fluid and the solid are required. This routines can cause the calculation of the coupling matrix to be slower than an implementation for only congruent meshes and with coupling elements in the interface where a lot of loops of elements are avoided. However, this increase of time is minimised if appropriate topological information is stored (i.e. tag the elements in order to minimise loop lengths or perform mappings). In addition, the cost of calculating a coupling matrix is often small when compared with the other tasks to be performed by the software and it is preferable to minimise the memory storage requirements. It is then important to avoid constraints when constructing the meshes of the problem. ACTRAN is an example of code dealing with both congruent and non-congruent meshes, according to [20].

Our implemetation of the FSM for vibroacoustic problems can deal with noncongruent meshes. A detail of the coupling zone in the calculation domain can be seen in Fig. 2. The continuous blue line represents the profile of the shell finite strips. The dashed light blue line represents the contour of the fluid domain that can be in contact with the shell finite strip. This contour is part of the fluid elements, in this case triangles. The integral required for the $\mathbf{L}^{F S}$ coefficients is calculated from the fluid side. The procedure described in Table 2 is followed.

To compute the integral from the fluid side has the advantage that situations with a solid immersed in a fluid can be handled with less difficulties. The typical example is a shell surrounded by fluids at both sides. It is then very important to check the orientation of the outward normal vector to the shell (or the local coordinates in the shell element) with respect to the outward normal vector to the acoustic domain. When the immersed solid is modelled by means of massive elements it is quite indifferent if the integral is performed following the fluid or solid contact contour and the checking of normal vector orientation can be avoided if meshes are generated with a consistent element orientation criterion. 


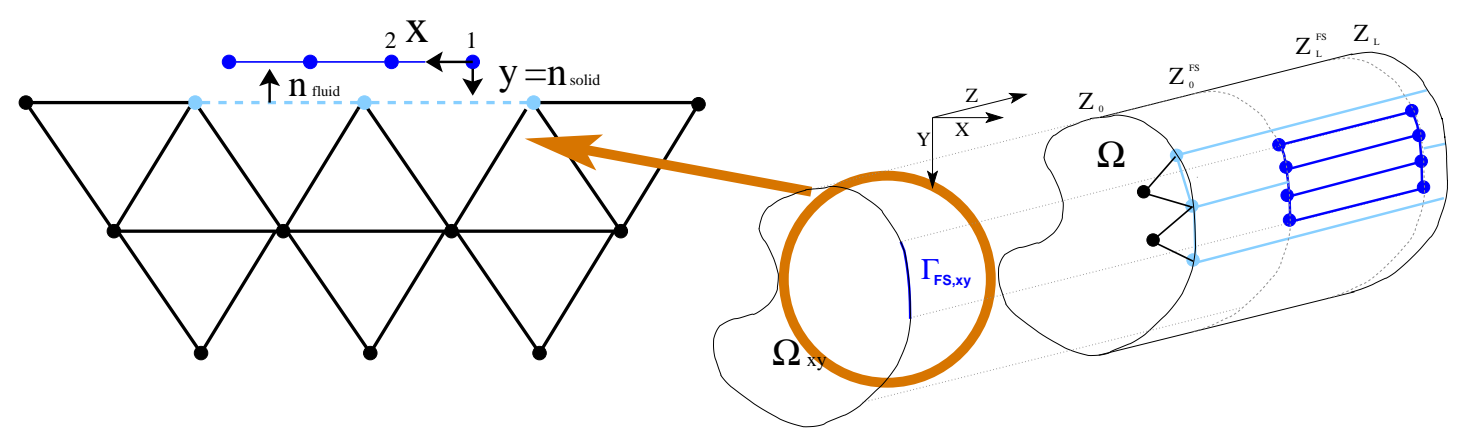

Figure 2: Sketch of the element interface in the coupling zone. On the right, physical (three-dimensional) and computational (two-dimensional) domains. A patch of three shell finite strips is represented. Two acoustic finite strip elements are in contact with this shell finite strips. On the left a detail of the coupling zone in the computational domain is shown.

Table 2: Procedure followed in order to calculate the fluid-solid interaction matrices in the two-dimensional calculation domain.

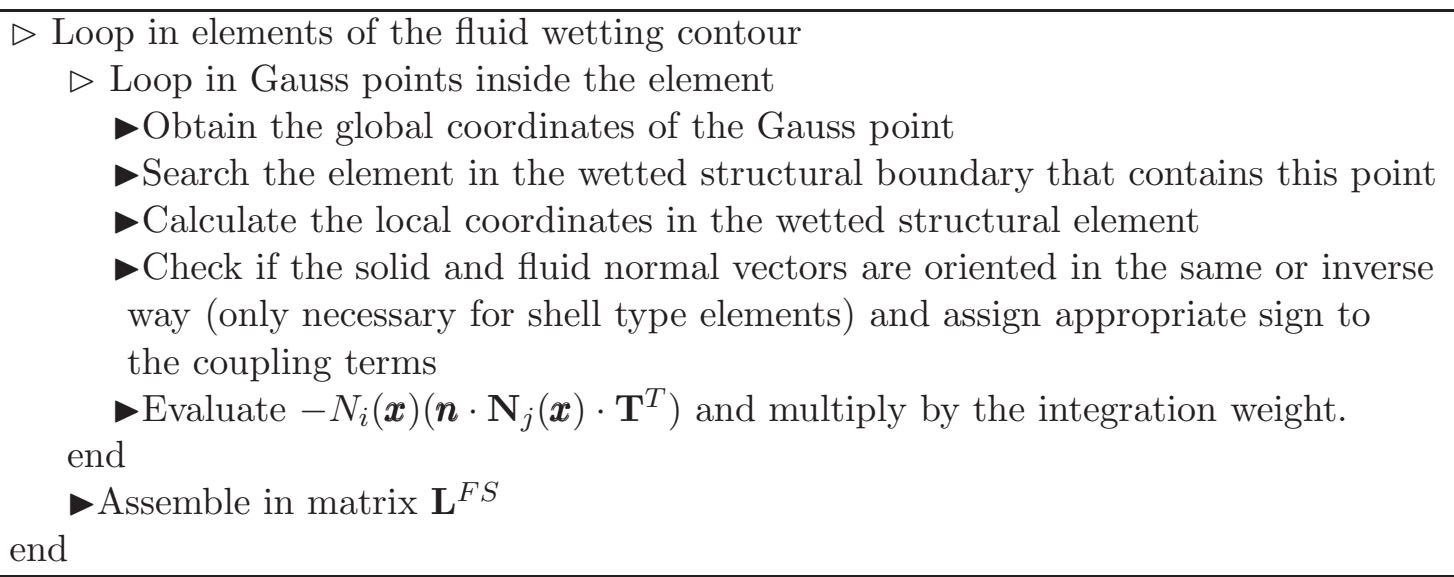

Another important aspect to take into account is to use an adaptive quadrature rule. The number of Gauss points must be increased, for example, if a large number of structural elements are in contact with a single fluid element. A geometrical tolerance has been established in order to decide if the Gauss point is in contact or not with the wetted structural contour.

\section{Application examples}

Various examples of the use of the FSM in acoustic and vibroacoustic problems will be shown in this section. A first group of examples deals with the frequency response of acoustic domains. An example with complex geometry (coupled acoustic cavities) illustrates the possibilities of the FSM for acoustic problems. In a second group of examples, the FSM is applied to the vibroacoustic problem. Comparisons of the FSM 
with the FEM have been done. The possibilities of the FSM for vibroacoustic problems are illustrated with a final example where the vibration transmission between the leaves of a double wall are predicted. The wall geometry is complex at cross-section level due to the stud shape. In addition the coupling between the leaves and the air cavity between them is high. This can be a problem for other modelling techniques that require weak coupling (which is not the case of the FSM).

\subsection{Frequency response of acoustic domains}

Two different cases have been considered to illustrate how the FSM can be used for acoustic problems. In order to obtain the pressure field in the acoustic domain for a single frequency, Eq. (15) must be solved for a finite number of $r$ and $s$ values $\left(r, s=0,1, \ldots, n_{Z}^{F}\right)$. The results are the $r^{t h}$-contribution to the pressure field, $\mathbf{p}_{r}$. These must be combined according to Eq. (11). In the first example of Section 3.1.1, the case of a point sound source in a cuboid shaped room is considered. This is a well known acoustic problem with an approximate expression of the pressure field. This expression is based on modal analysis and can be obtained without additional software. Finally, an example with a more complex extrusion geometry that illustrates the capabilities of the FSM for realistic problems is presented in Section 3.1.2.

Acoustic absorption is modelled by means of Robin boundaries. The admittances considered are shown in Table 3.

Table 3: Values of normalised admittance and averaged absorption for the Robin boundary condition.

\begin{tabular}{cccc}
$1 / A \rho_{\mathrm{F}} c$ & 6 & 20 & 70 \\
\hline$\alpha(\%)$ & 51 & 18 & 6
\end{tabular}

\subsubsection{Comparison with modal analysis: point source in a cuboid shaped room}

A cuboid shaped room with dimensions $L_{x}=2 \mathrm{~m}, L_{y}=3 \mathrm{~m}$ and $L_{z}=3.5 \mathrm{~m}$ and a point sound source (with a source strength $q_{s}=0.015 \mathrm{~m}^{3} / \mathrm{s}$ ) placed at position $X_{s}=0.3 \mathrm{~m}, Y_{s}=0.4 \mathrm{~m}, Z_{s}=0.6 \mathrm{~m}$ has been considered. Direction $z$ has been chosen to be the extrusion direction. The output is the sound pressure level of the room, evaluated as

$$
L=10 \log _{10}\left(\frac{\left\langle p_{\text {rms }}^{2}\right\rangle}{p_{0}^{2}}\right) \quad \text { with }\left\langle p_{\text {rms }}^{2}\right\rangle=\frac{1}{n_{\text {nodes }}^{F}} \sum_{i=1}^{n_{\text {nodes }}^{F}} \frac{\left|p_{i}\right|^{2}}{2}
$$

where $\left\langle p_{\text {rms }}^{2}\right\rangle$ is the space averaged root mean square pressure. In the FSM it is calculated by averaging the nodal values $p_{i}$ of 20 equally spaced cross-sections (each cross-section has a constant $z$ coordinate). $n_{\text {nodes }}^{F}$ nodal values are used for each crosssection. The values of acoustic absorption considered are shown in Table 3. 
An approximate solution of the problem can also be obtained by means of analytical modal analysis (see for example [21, 22]). This analytical formulation provides a more accurate pressure field for the case without acoustic absorption. The results are shown in Fig. 3.

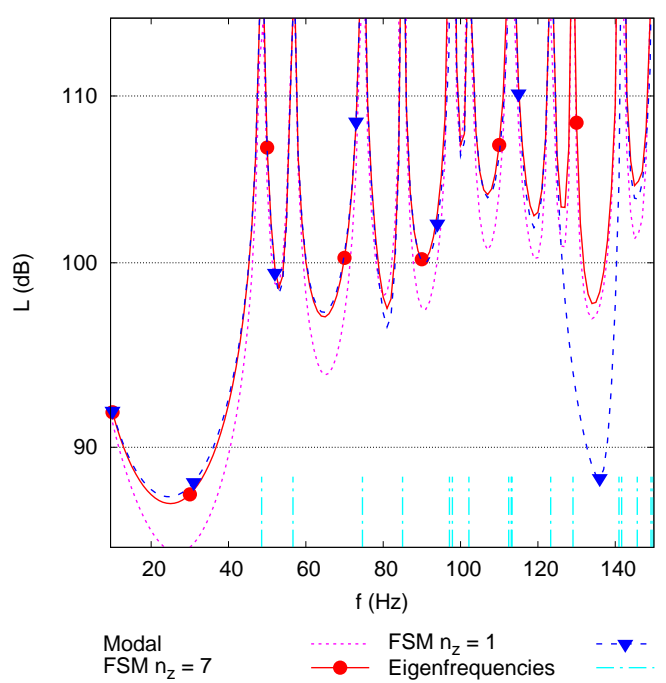

(a)

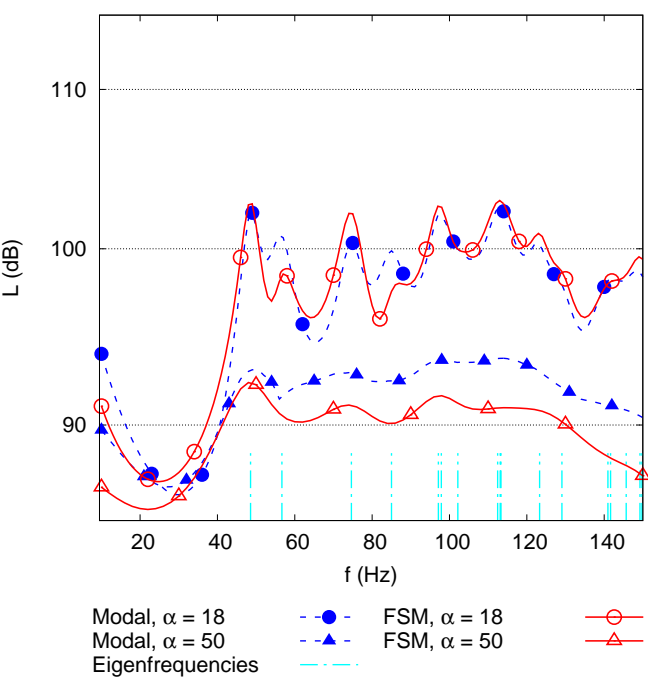

(b)

Figure 3: Comparison between the pressure fields obtained by means of the FSM and the analytical modal analysis: (a) no acoustic absorption; (b) with acoustic absorption.

Only the lower frequencies have been considered. For higher frequencies the modal density is too large and the results too diffuse in order to understand the performance of the numerical method. In any case the FSM can be used in order to decrease the computational costs for a fixed element size and, for the same problem, be able to calculate higher frequencies.

The set of functions $\mathcal{Z}_{r}(z)$ has been truncated for a value of $r>2 f_{\max } L_{z} / c$. This value ensures that even the pressure field which is the most similar to the eigenmode with a maximum number of waves in the $z$ direction (constant pressure field in the $X Y$ plane) is correctly described. Unfortunately, the impossibility of a priori discarding some functions of the set is a drawback of the FSM when performing a frequency response analysis. The shape of the expected pressure field is unknown a priori due to the high modal density and the large number of frequencies of interest (it is common to perform a large number of calculations in a frequency band). The vector $\mathbf{p}_{r}$ with a more important contribution to the pressure field for a fixed frequency is not known a priori and the interpolation field must be prepared for all the possible modal shapes (all the possible values of $r$ ).

An example of numerical error caused by a poor set of interpolation functions in the extrusion direction can be found in Fig. 3(a). The case where only two interpolation functions in the $z$ direction are used cannot provide good results around the frequency $129.08 \mathrm{~Hz}$. This is an eigenfrequency with mode $\left(n_{x}=1, n_{y}=0, n_{z}=2\right)$, isolated from other eigenfrequencies of the domain. The pressure field clearly has a wave in 
the $z$ direction and cannot be described by the functions $\mathcal{Z}_{0}(z)$ (constant along the $z$ direction) and $\mathcal{Z}_{1}(z)$ (half wave along the $z$ direction, see Eq. (27)). At least $\mathcal{Z}_{2}(z)$ is required.

The agreement between both approaches is better for smaller values of the acoustic absorption. The FSM interpolation field can satisfy the Robin boundary condition. This is not the case of pressure fields interpolated with modal functions obtained by assuming purely reflecting boundaries.

\subsubsection{Sound propagation between coupled acoustic domains}

The FSM has been used to solve a more complex problem without analytical solution. Three acoustic domains, coupled as shown in the plan of Fig. 4, have been considered (they are like coupled rooms [23]). The values $L_{x}=5 \mathrm{~m}, L_{y}=3 \mathrm{~m}, L_{z}=2.5$ $\mathrm{m}, \epsilon=0.25, \beta=0.4$ and $\delta=0.05$ remain constant along the analysis. A point sound source at the position $X_{s}=1.49 \mathrm{~m}, Y_{s}=1.48 \mathrm{~m}, Z_{s}=1 \mathrm{~m}$ with a source strength $q_{s}=0.015 \mathrm{~m}^{3} / \mathrm{s}$ is considered. The sound source is placed in the sending room that is connected with the receiving room. The goal of the problem is to study how the sound level difference between the sending and the receiving rooms ( $D=L_{\text {sending }}-L_{\text {receiving }}$ ) depends on the openings width and the acoustic absorption of the walls. $L$ is calculated inside each room according to Eq. (46).

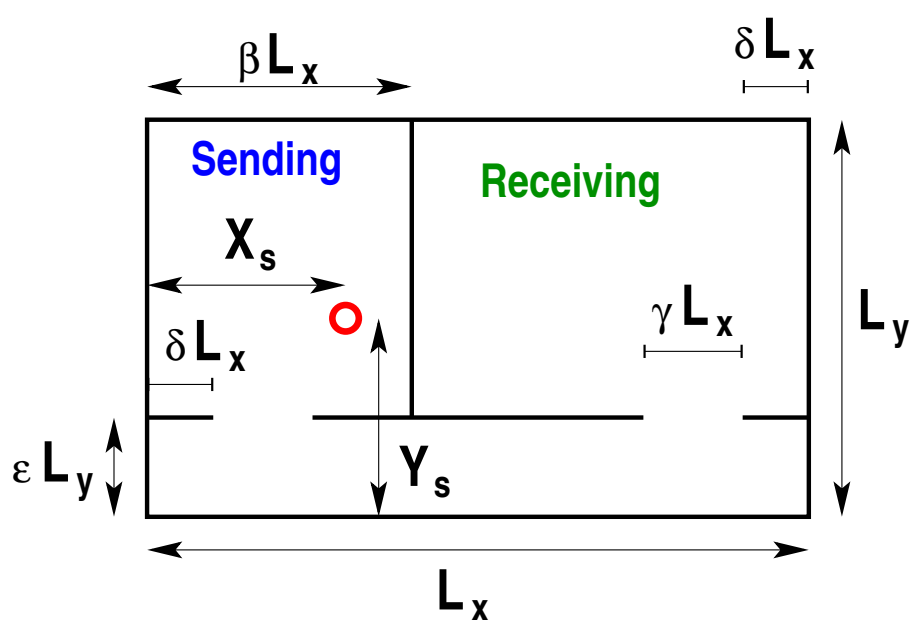

Figure 4: Building plan with three coupled rooms. A point source is placed in the sending room.

Note that the extrusion symmetry direction is orthogonal to the plan. Robin boundary conditions with the admittances shown in Table 3 are imposed at the vertical surfaces. Interpolation functions defined in Eq. (27) are used, thus the floors and the ceilings of the rooms are purely reflecting. A width of $0.1 \mathrm{~m}$ has been given to all the vertical partitions. However, this is only used to build the mesh. This is an acoustic (not vibroacoustic problem) and sound transmission through the partitions is not considered. Thus, the sound level difference shown as output corresponds to an acoustic path through the openings. 
For frequencies below $500 \mathrm{~Hz}$, one calculation each $\mathrm{Hz}$ and eight functions $\left(n_{Z}^{F}=8\right)$ in the $z$ direction have been used. For frequencies over $500 \mathrm{~Hz}$, one calculation each five $\mathrm{Hz}$ and $n_{Z}^{F}=22$ functions in the $z$ direction have been used. In both cases quadratic triangles have been used in the $X Y$ plane with mesh sizes of $0.15 \mathrm{~m}$ and $0.055 \mathrm{~m}$ respectively.

The dependence of the sound level difference $D$ on the opening width $\gamma L_{x}$ and the acoustic absorption of the walls is shown in Fig. 5. A third octave band average has been done. In the low-frequency range, the influence of the opening width is small. The global behaviour highly depends on the resonances of the problem and modes increasing the pressure level in both rooms can be found in each frequency band. On the contrary, the opening width is an important parameter for frequencies above $100 \mathrm{~Hz}$. The sound level difference is higher for the cases with larger room connection. The acoustic absorption also plays an important role. It mainly amplifies the differences between cases with different opening widths.

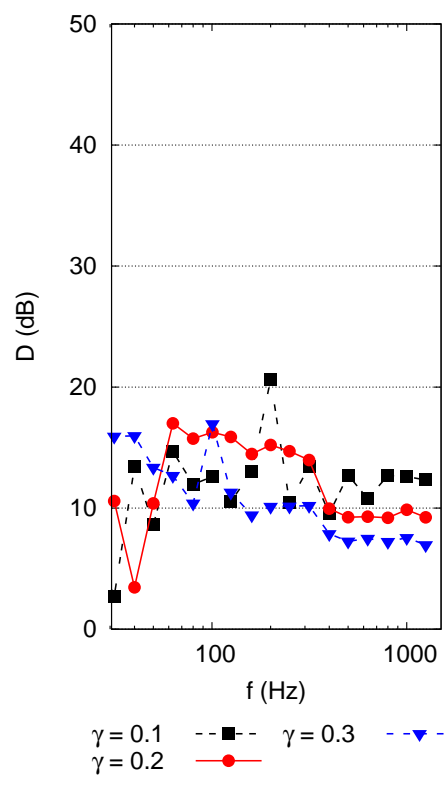

(a)

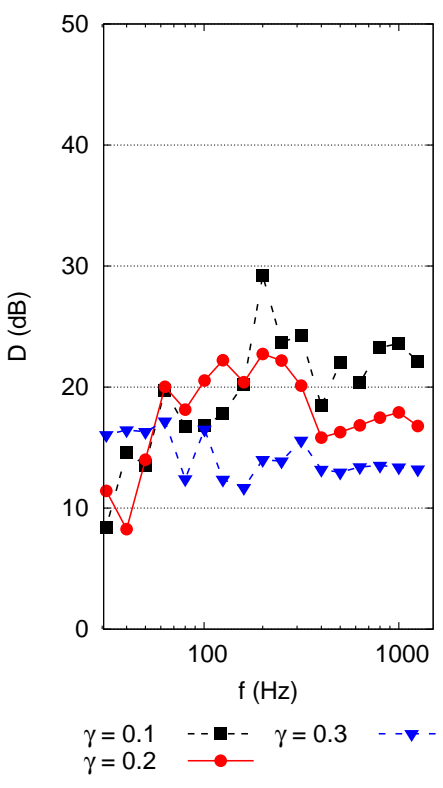

(b)

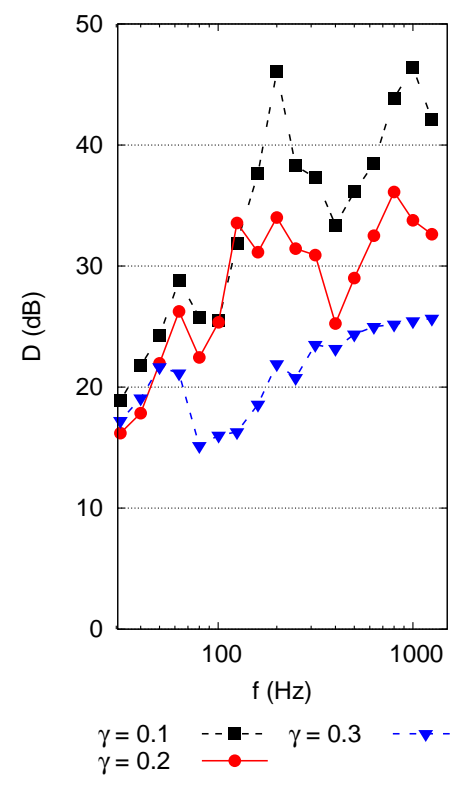

(c)

Figure 5: Sound level difference between the sending and receiving rooms for several values of acoustic absorption $\alpha$ and opening width $\gamma$ : (a) $\alpha=6 \%$; (b) $\alpha=18 \%$; (c) $\alpha=50 \%$.

\subsection{Vibroacoustic problems}

\subsubsection{Comparison with the FEM: pressure excited plate}

The FSM solution of vibroacoustic problems has been compared with the FEM solution provided by Code-Aster. This is available on line [18] and can be used under free software licenses. As detailed in Section 2.1 the pressure-displacement formulation has been considered in our implementation of the FSM. In Code-Aster the pressure and 
the displacement potential is considered in order to describe the fluid. This doubles the number of degrees of freedom in the fluid but leads to a symmetric formulation of the vibroacoustic problem. More details can be found in [24]. The information on its acoustic FEM part can be found in [25]. In any case, the pressure and displacement fields obtained should be the same for both approaches.

The model problem considered to compare the FSM and the FEM results is the cavity of Section 3.1.1 but with a rectangular plate $\left(L_{y}=3 \mathrm{~m} \times L_{z}=3.5 \mathrm{~m}\right)$ with the displacements blocked along its boundary (see Fig. 6 where the structure boundary is identified as $\left.\Gamma_{F S}\right)$. The geometrical and mechanical properties of the wall can be found in Table 4. The DKT shell element is used in Code-Aster and a rectangular shell strip with cubic polynomial interpolation for the out-of-plane displacements and linear interpolation for the in-plane displacements is used in the FSM model. The excitation is an imposed velocity in the $\Gamma_{N}$ square surface $(0.2 \mathrm{~m} \times 0.2 \mathrm{~m}$ with $L_{N, y}=L_{N, z}=0.5 \mathrm{~m}$ according to Fig. 6). Thus, we can see how the FSM only requires extrusion symmetry in the geometry but not in the boundary conditions. An acoustic absorption of $18 \%$ according to Table 3 is considered in the boundary $\Gamma_{R}$.

Table 4: Material and geometrical properties of the concrete plate.

\begin{tabular}{lll} 
Meaning & Symbol & Value \\
\hline Young's modulus & $E$ & $2.94 \cdot 10^{10} \mathrm{~N} / \mathrm{m}^{2}$ \\
Solid density & $\rho_{\mathrm{S}}$ & $2500 \mathrm{~kg} / \mathrm{m}^{3}$ \\
Wall thickness & $t$ & $0.1 \mathrm{~m}$ \\
Poisson's ratio & $\nu$ & 0.25 \\
Hysteretic damping coefficient & $\eta$ & $0 \%$ \\
\hline
\end{tabular}

The outputs considered are the pressure phasor at the position $(0,0,0)$ and the normal displacement $\left(U_{n} \equiv U_{X}\right)$ to the plate point $(2,2.25,3)$. The reference value is taken from a FSM calculation with linear finite element size $h=0.03 \mathrm{~m}$ both in the acoustic domain and in the plate with the following interpolation in the $z$ direction: $n_{Z}^{F}=10$ (acoustic domain) and $n_{Z}^{S}=5$ (structure).

The relative difference between the FEM solution and the reference FSM solution for the fluid and the solid are calculated as

$$
e_{F}=\frac{\left|p_{h}-p_{\mathrm{ref}}\right|}{\left|p_{\mathrm{ref}}\right|} \quad e_{S}=\frac{\left|\left(U_{n}\right)_{h}-\left(U_{n}\right)_{\mathrm{ref}}\right|}{\left|\left(U_{n}\right)_{\mathrm{ref}}\right|}
$$

In the vibroacoustic problem, the dependence of computational costs on the implementation and chosen algorithms is larger than for the case of the acoustic problem. The use of a direct solver for banded matrices is not a reasonable option if realistic problems have to be solved for low and mid frequencies. The number of operations required by the direct solvers for sparse matrices depends a lot on the implementation and the topology of the domains discretisation (including the contacts between the fluid and the solid). It is, consequently, very problem dependent. Moreover, strategies that can help with the optimisation of the resolution by considering the degree of coupling between fluid and solid domains can be considered (see, for example, [26]). 


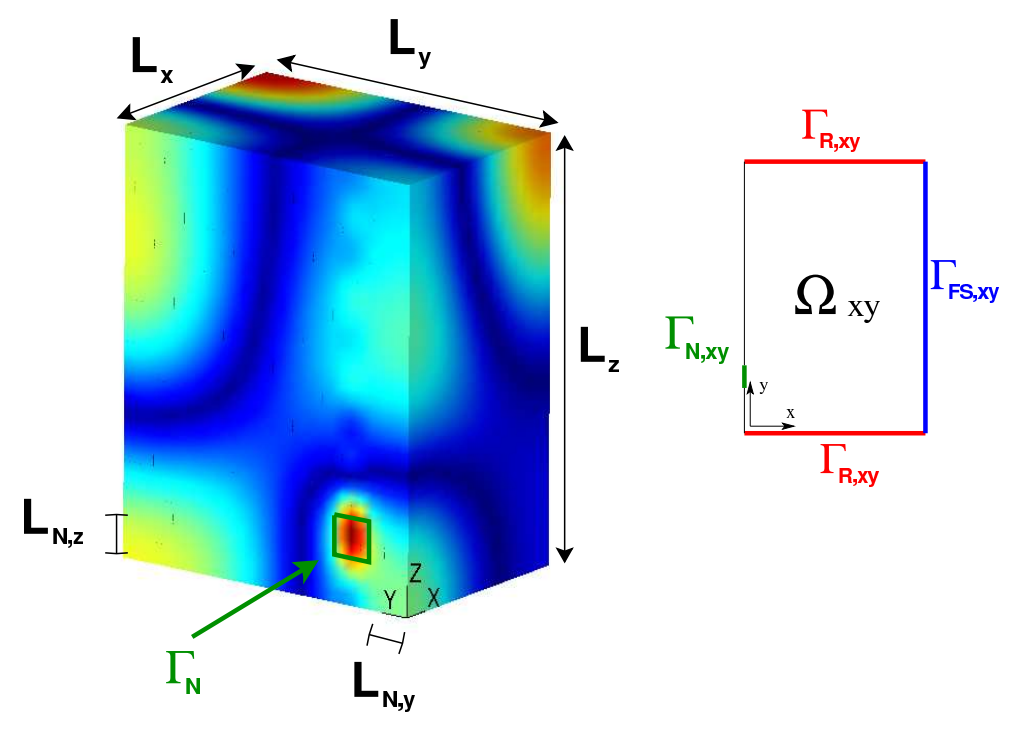

Figure 6: Sketch of the example used to compare the FSM with the FEM in vibroacoustic problems. The three-dimensional background image of the pressure field corresponds to a frequency of $110 \mathrm{~Hz}$ in a problem solved by means of the FSM.

In cases with weak coupling, staggered strategies where the fluid and solid domains are solved independently and the coupling effect is taken into account a posteriori by means of iterative corrections can be considered. When this happens, the solution of the coupled linear system of equations can be avoided. For these reasons the numerical error has been plotted depending on the number of degrees of freedom of the problem in Fig. 7.

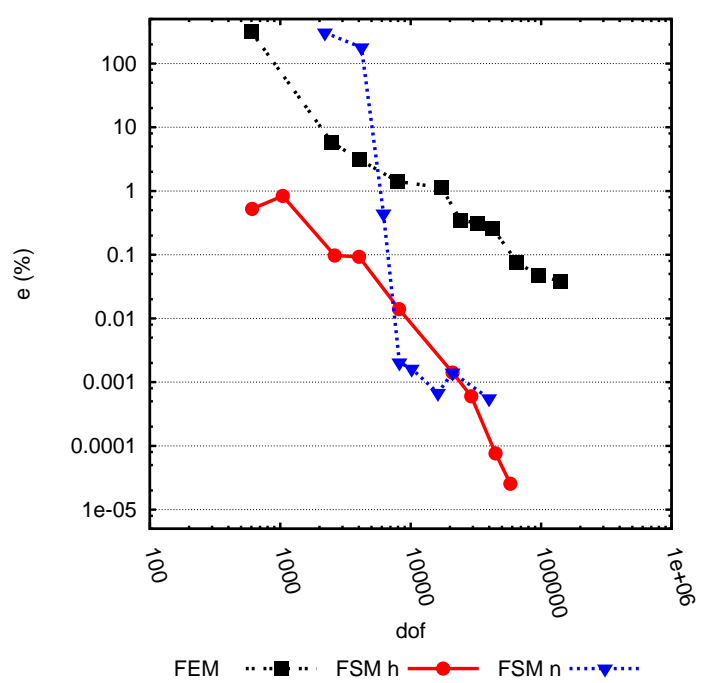

(a)

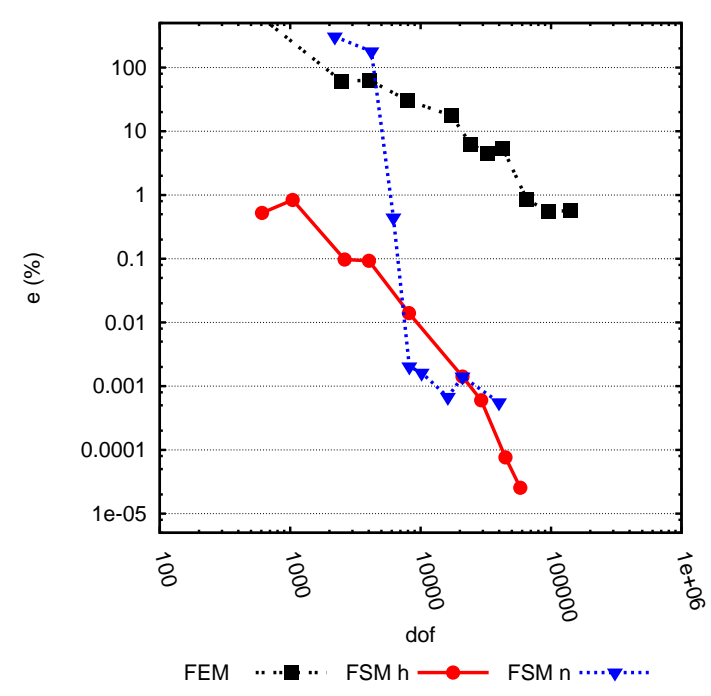

(b)

Figure 7: Comparison between the FSM and the FEM for a vibroacoustic problem (the frequency is $165 \mathrm{~Hz}$ ) : (a) displacement; (b) pressure. 
Three different refinement options have been considered: (i) The FEM congruent mesh is refined (with element lengths in a range between $0.5 \mathrm{~m}$ and $0.04 \mathrm{~m}$ ); (ii) 'FSM h', the solution is obtained with the FSM. The two-dimensional mesh is refined (with element lengths in a range between $0.5 \mathrm{~m}$ and $0.035 \mathrm{~m}$ ) while the number of functions used for the extrusion direction is kept constant (and large enough for the frequency of interest with $n_{Z}^{F}=10$ and $n_{Z}^{S}=5$ ); (iii) 'FSM n', the solution is obtained with the FSM. The two-dimensional mesh has a constant element size $(0.06 \mathrm{~m})$ while the number of functions used for the extrusion direction is progressively increased $\left(n_{Z}^{F}=0,1,2,3,4,7,10,20\right.$ and $n_{Z}^{S}=1,2,3,4,5,8,5,10$ respectively).

It can always be seen how for the same numerical error, the computational costs for the FSM are significantly smaller than for the FEM. The error of the FSM solution is always two orders of magnitude smaller than the FEM error for a given number of degrees of freedom. In the FSM, the solution can be improved by refining the twodimensional mesh and increasing the number of functions for the extrusion direction. To perform only one of the two actions is not enough as shown by the case 'FSM n' where, after an over-abundant increase of $n_{Z}^{F}$, the numerical error remains constant.

\subsubsection{Double wall analysis}

A lightweight double wall is an example of vibroacoustic system with extrusion symmetry. The cross-section usually has a quite complex geometry that is extruded along a line. In addition, the coupling between the cavities and the structure is strong. This invalidates most of the one-way-coupling hypotheses assumed in simplified vibroacoustic models and the use of numerical techniques such as the FEM or the FSM becomes more important.

The example shown here is based on the lightweight double walls studied in [27]. The role of the studs in the vibration transmission between leaves was studied at crosssection level by means of two-dimensional models. Now, the vibration transmission between the leaves has been predicted by means of a FSM three-dimensional analysis. The coupling with the air cavities is now considered (it was omitted in [27] in order to simplify the problem and focus the analysis in the structural path of vibration transmission). The coupled problem is significantly expensive and this limits the frequency range that can be analysed.

A double wall with four C-shaped studs has been considered. The dimensions of the double wall are $0.07 \mathrm{~m}$ (height of the cavity) $\times 3.0 \mathrm{~m} \times 3.5 \mathrm{~m}$ (extrusion direction). The separation between the studs is $0.6 \mathrm{~m}$. A sketch of the double wall cross-section and the stud can be found in Fig. 8. The dimensions of the stud according to the notation in Fig. 8 are: $h=7 \mathrm{~mm}, b=4 \mathrm{~mm}$ and $c=1 \mathrm{~mm}$ with a thickness of $0.47 \mathrm{~mm}$. The studs and the leaves are connected along a line. The geometrical and mechanical properties of the leaves can be shown in Table 5 .

A pressure is applied in a $0.1 \mathrm{~m} \times 0.1 \mathrm{~m}$ square at a distance of $1.2 \mathrm{~m}$ of the border of the double wall. The vibration level difference between the leaves of the double wall is calculated as

$$
D_{\nu, i j}=10 \log _{10}\left(\frac{<\left|u_{\text {upper }}\right|^{2}>}{<\left|u_{\text {lower }}\right|^{2}>}\right)
$$


where $u_{\text {upper }}$ and $u_{\text {lower }}$ are the phasors of displacements for the upper and lower leaves. The spatial average is done along the leave. A larger value of $D_{\nu, i j}$ means a better vibration isolation.

Table 5: Geometrical and mechanical properties of the leaves

\begin{tabular}{lll} 
Meaning & Symbol & Value \\
\hline Thickness & $t$ & $13 \mathrm{~mm}$ \\
Young's modulus & $E$ & $2.5 \cdot 10^{9} \mathrm{~N} / \mathrm{m}^{2}$ \\
Density & $\rho_{\mathrm{S}}$ & $692.3 \mathrm{~kg} / \mathrm{m}^{3}$ \\
Damping & $\eta$ & $3 \%$ \\
\hline
\end{tabular}

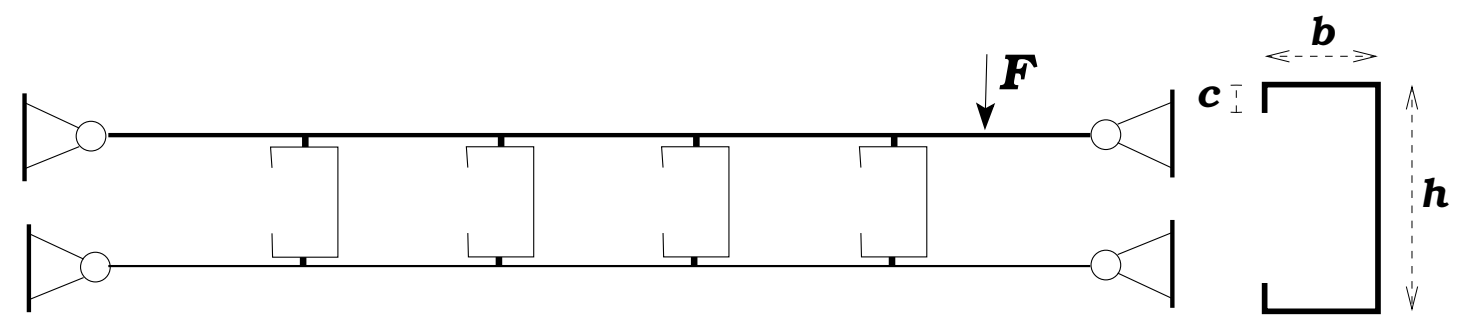

(a)

(b)

Figure 8: Sketch of the double wall, the stud axis follows the extrusion direction: (a) $X Y$ cross-section; (b) C-shaped stud.

Three different situations have been considered:

1. A simple double wall composed of the two leaves and an air cavity between them

2. A structure composed by the two leaves and the C-shaped studs

3. The 'real' double wall composed of the two leaves, and four C-shaped studs linking them. The cavity between leaves is divided into five channels by the studs

The first model is used to estimate the vibration transmission through the cavity (cavity path). The vibration in the upper leave is transmitted to the air cavity and the pressure in the cavity generates vibration in the lower leave. The goal of the second situation is to show how the vibrations are transmitted through the structure in absence of acoustic cavities (stud path). Finally, a realistic model of the double wall including all the transmission paths between leaves is considered in order to show how are they balanced in the double wall.

The results are shown in Fig. 9. The cavity path is more important for frequencies below $175 \mathrm{~Hz}$. Above this frequency, the most important part of the vibrations are transmitted through the structure. The 'real' situations represents a lower bound of both transmission paths (cavity and stud). The isolation of vibrations increases with 
frequency. It is clear that, even for the case of air cavities (not filled with absorbing materials), the insulation capacity of these lightweight double walls is controlled by the flexibility of the studs.

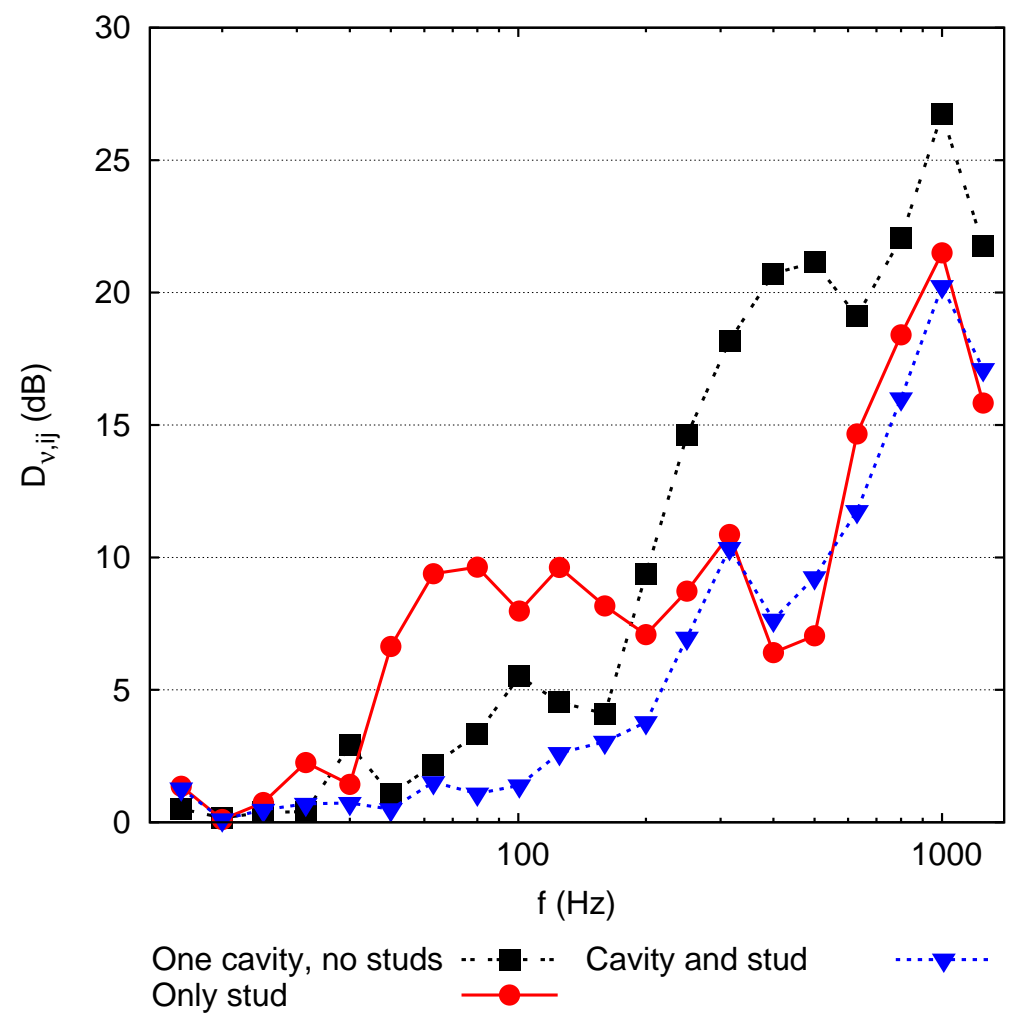

Figure 9: Vibration level difference between the leaves of a double wall for several wall configurations.

\section{Conclusions}

A finite strip formulation for acoustic and vibroacoustic problems (pressure-displacement formulation) has been presented. This technique has been used in order to solve problems in finite dimension domains with Neumann (purely reflecting) and Robin (absorbing) boundary conditions in the extrusion direction. A set of trigonometric functions (strip functions) is considered in the extrusion direction in order to interpolate the pressure and displacement fields. This is necessary in order to describe the modal response of the system and reproduce phenomena such as the resonant transmission of sound (important in building acoustics). This cannot be modelled if the wave length along the extrusion direction is imposed a priori.

Some finite strip functions for the acoustic problem have been proposed. Only in the case of purely reflecting boundary conditions in the extrusion direction the contribution of each strip function can be obtained independently from the others. In general, if absorption is considered, a coupled linear system of equations is obtained. 
This represents a disadvantage of the acoustic FSM if compared with the structural FSM. In the vibroacoustic problem the coupling between the acoustic and solid strip function contributions cannot, in general be avoided because: (i) different strip functions are used in the acoustic and solid parts of the problem in order to satisfy the boundary conditions; (ii) the dimensions of the solid and the fluid domains along the extrusion direction can be different.

Various comparisons of the FSM results with the FEM and with analytical modal analysis have been done. The FEM results has been generated by means of CodeAster. The agreement with the FSM is good for both acoustic and vibroacoustic problems. In the comparison with modal analysis the agreement is also correct. Especially for cases with low acoustic absorption.

The computational costs of solving a three-dimensional problem with the FSM are significantly smaller than the FEM costs. This can be used in order to increase the frequency range modelled with a deterministic model.

The FSM is better than other numerical models that use trigonometric functions to reduce the computational costs (such as the acoustic modal analysis) in order to reproduce the boundary conditions of the problem. The use of polynomial interpolation at cross-section level implies that the normal derivative of the pressure field at the contour can be non-null. This is important in order to satisfy the absorbing boundary conditions (very relevant and used in building acoustics) and the coupling with the structure.

The FSM represents an useful tool to deal with building acoustic problems with an extrusion symmetry geometry that is complex enough at cross-section level to make very difficult the formulation of analytical models (like for example Section 3.2.2).

\section{Acknowledgements}

Free software has been used [18, 28-34].

\section{References}

[1] Y.K. Cheung and L.G. Tham. Finite strip method. CRC Press, 1997.

[2] R. Friedrich. Finite strip method: 30 years. Eng. Comput., 17(1):92-111, 2000.

[3] S. Finnveden. Evaluation of modal density and group velocity by a finite element method. J. Sound Vibr., 273(1-2):51-75, 2004.

[4] S. Finnveden and M. Fraggstedt. Waveguide finite elements for curved structures. J. Sound Vibr., 312(4-5):644-671, 2008.

[5] V. Damljanović and R.L. Weaver. Propagating and evanescent elastic waves in cylindrical waveguides of arbitrary cross section. J. Acoust. Soc. Am., 115(4):1572-1581, 2004.

[6] U. Orrenius and S. Finnveden. Calculation of wave propagation in rib-stiffened plate structures. J. Sound Vibr., 198(2):203-224, 1996. 
[7] C.M. Nilsson and S. Finnveden. Input power to waveguides calculated by a finite element method. J. Sound Vibr., 305(4-5):641-658, 2007.

[8] R.J. Astley and A. Cummings. A finite element scheme for acoustic transmission through the walls of rectangular ducts: comparison with experiment. J. Sound Vibr., 92(3):387-409, 1984.

[9] R.J. Astley, A. Cummings, and N. Sormaz. A finite element scheme for acoustic propagation in flexible-walled ducts with bulk-reacting liners, and comparison with experiment. J. Sound Vibr., 150(1):119-138, 1991.

[10] C.M. Nilsson and S. Finnveden. Waves in thin-walled fluid-filled ducts with arbitrary cross-sections. J. Sound Vibr., 310(1-2):58-76, 2008.

[11] G.C. Everstine. Finite element formulations of structural acoustics problems. Comput. Struct., 65(3):307-321, 1997.

[12] J.P. Morand and R. Ohayon. Fluid-Structure Interaction: Applied Numerical Methods. Masson - Wiley, Chichester; New York; Paris; 1995.

[13] N. Atalla and R.J. Bernhard. Review of numerical solutions for low-frequency structural-acoustic problems. Appl. Acoust., 43:271-294, 1994.

[14] F. Ihlenburg. Finite element analysis of acoustic scattering. Springer, 1998.

[15] S. Maluski and B.M. Gibbs. Application of a finite-element model to lowfrequency sound insulation in dwellings. J. Acoust. Soc. Am., 108(4):1741-1751, 2000 .

[16] J.P. Coyette. The use of finite-element and boundary-element models for predicting the vibro-acoustic behaviour of layered structures. Adv. Eng. Softw., 30:133-139, 1999.

[17] O.C. Zienkiewicz and R.L. Taylor. The finite element method. ButterworthHeinemann, 2000.

[18] EDF. Code-Aster home page. http://www.code-aster.org, 2010.

[19] C.M. Nilsson. Waveguide finite elements applied on a car tyre. PhD thesis, KTH, Royal institute of technology (Department of Aeronautical and Vehicle Technology), 2004.

[20] A. Genard et al. Actran 2005 user's manual. Free Field Technologies, 2005.

[21] A.D. Pierce. Acoustics. An introduction to its physical principles and applications. McGraw Hill, New York, 1981.

[22] T.E. Vigran. Building acoustics. Taylor and Francis, London, 2008.

[23] C.M. Harris and H. Feshbach. On the acoustics of coupled rooms. J. Acoust. Soc. Am., 22(5):572-578, 1950. 
[24] N. Greffet. Éléments vibro-acoustiques. EDF(http://www.codeaster.org/V2/doc/default/man_r/r4/r4.02.02.pdf), 2009.

[25] C. Durand. Éléments finis en acoustique. EDF (http://www.codeaster.org/V2/doc/default/man_r/r4/r4.02.01.pdf), 2009.

[26] J. Poblet-Puig and A. Rodríguez-Ferran. The block Gauss-Seidel method in sound transmission problems. J. Comput. Acoust., 1(18):13-30, 2010.

[27] J. Poblet-Puig, A. Rodríguez-Ferran, C. Guigou-Carter, and M. Villot. The role of studs in the sound transmission of double walls. Acta Acust. United Acust., 45(3):555-567, 2009.

[28] C. Geuzaine and J.-F. Remacle. Gmsh: a three-dimensional finite element mesh generator with built-in pre- and post-processing facilities. Int. J. Numer. Meth. Engng., 11(79):1309-1331, 2009.

[29] S. Balay, K. Buschelman, W.D. Gropp, D. Kaushik, M.G. Knepley, L.C. McInnes, B.F. Smith, and H. Zhang. PETSc Web page, 2001. http://www.mcs.anl.gov/petsc.

[30] S. Balay, K. Buschelman, W.D. Eijkhout, V. Gropp, D. Kaushik, M.G. Knepley, L.C. McInnes, B.F. Smith, and H. Zhang. PETSc users manual. Technical Report ANL-95/11 - Revision 2.1.5, Argonne National Laboratory, 2004.

[31] S. Balay, W.D. Gropp, L.C. McInnes, and B.F. Smith. Efficient management of parallelism in object oriented numerical software libraries. In E. Arge, A. M. Bruaset, and H. P. Langtangen, editors, Modern Software Tools in Scientific Computing, pages 163-202. Birkhäuser Press, 1997.

[32] V. Hernandez, J.E. Roman, and V. Vidal. SLEPc: A scalable and flexible toolkit for the solution of eigenvalue problems. ACM Transactions on Mathematical Software, 31(3):351-362, September 2005.

[33] V. Hernandez, J.E. Roman, and V. Vidal. SLEPc: Scalable Library for Eigenvalue Problem Computations. Lecture Notes in Computer Science, 2565:377-391, 2003.

[34] J. E. Roman, E. Romero, and A. Tomas. SLEPc home page. http://www.grycap.upv.es/slepc, 2010. 